\title{
Development of classical models for the description of QM properties in photoinduced processes: the case of peridinin
}

Ingrid G. Prandi ${ }^{1 *}(\mathrm{PG})$, Benedetta Mennucci ${ }^{2}$

1 Department of Chemistry, Federal University of Lavras, 37200-000, Lavras, Brazil

2 Department of Chemistry, University of Pisa, Via Giuseppe Moruzzi 3, I-56124 Pisa, Italy

Corresponding author: ingrid.prandi@gmail.com

Keywords: Force-field, Molecular Dynamics, Carotenoid, Peridinin

\section{Introduction:}

Carotenoids are naturally occurring pigments present in photosynthetic organisms, such as plants, bacteria and algae. Specifically, peridinin (PID) is found in Light-Harvesting (LH) complexes where it play an important role acting as antennae, enhancing the light absorption for photosynthetic processes ${ }^{1}$. A common methodology to investigate the mechanisms of action of carotenoids in these complexes is the combination of quantum mechanics (QM) methods and classical molecular dynamics (MD) to simulate the system at in vivo conditions. Unfortunately, for an accurate prediction of the structure and their temperature-dependent fluctuations, it is not sufficient to describe carotenoids with a general force-field, but it is necessary to develop specialized force-field parameters. In this work, peridinin classical molecular dynamics simulations are performed with a new set of parameters and compared with quantum mechanical counterparts.

\section{Methodology:}

The starting point of the development of the new set of parameters was the geometry optimization of peridinin performed at B3LYP/6-311G(d,p) level of theory performed by GAUSSIAN $09^{2}$ package. Bonds and angles force constants were derived by $\mathrm{JOYCE}^{3}$ software from the diagonalization of the Hessian matrix. Dihedrals parameters were modeled by harmonic potentials or extracted from torsional energy profiles by the performance of a QM relaxed scan, depending on the stiffness of the dihedral torsion. Instead, for intramolecular parameters, we explicitly set to zero all of the Coulomb terms, while only some van der Walls interactions, between specific atoms, were taken into account. In such way, we have transferred all the intramolecular electrostatic effects to the force constants.

In order to test the quality of our results we have performed two different MD simulations: a correlated and an uncorrelated MD. For the validation of the model, all peridinin MD simulations were carried out with GROMACS ${ }^{4}$ software. For the analyses, we have extracted frames from the MD trajectories and performed geometric measurements and QM calculations on those geometries.

\section{Results and Discussion:}

The geometric analysis performed for the new peridinin force-field was the measure of the lengths of the conjugated bonds in its backbone. This analysis compares the bond lengths in DFT minimum structure and the average of the geometries taken from the uncorrelated MD. As a result, the classical model not only reproduces the trend in 
bond lengths but also their absolute values, presenting a very good agreement between DFT and classical approaches. We note that a very accurate description of the geometry is needed in order to get a proper description of the electronic properties, which, in the case of peridinin, are strongly affected by structural distortion, especially by the ones located in the backbone.

In order to verify the excitation properties, we performed TDA-B3LYP/6$311 \mathrm{G}(\mathrm{d}, \mathrm{p})$ calculations on 1000 geometries extracted from the uncorrelated MD trajectory and compared with the DFT optimized structure. In a general view, the results suggest that, in average, the new force-field describes the peridinin in a similar way to what predicted by a DFT geometry optimization.

In order to check the quality of the fitted constants and the vibrational modes, we have simulated two infrared (IR) spectra based on: a) B3LYP/6-311G(d,p) optimized structure, and b) MD geometries extracted from the simulation. In the latter case, the calculated vibrational spectra was obtained as the Fourier transform of molecular dipole moments autocorrelation function ${ }^{5}$.

The two spectra show an overall good agreement in the position of the peaks especially for those with frequencies higher than $800 \mathrm{~cm}^{-1}$. At low frequencies, differences are more evident; this can be explained observing that during the classical MD, the molecule can sample conformations, mainly dihedrals and out of plane motions, far from the minimized geometry.

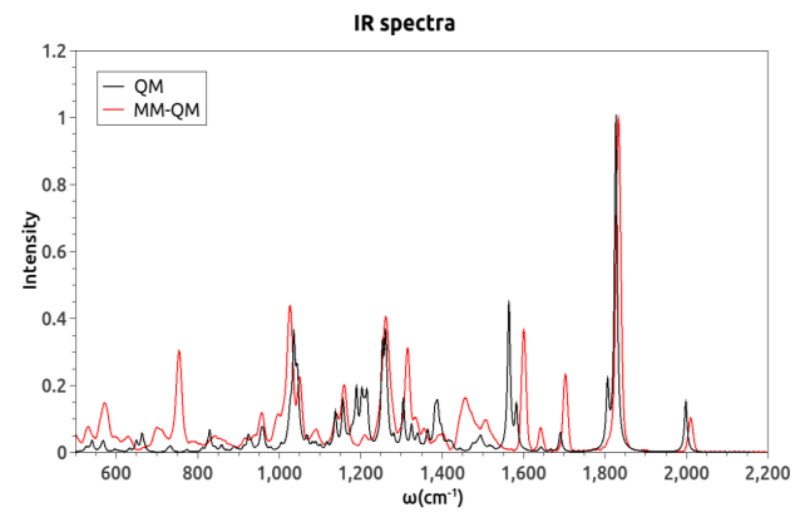

\section{Conclusions}

With this work we can conclude that despite the current limitations of the classical model, it is possible to recover much of the electronic effects lost in a classical MD by just modelling the molecule of interest with a very specific set of parameters.

\section{Acknowledgments:}

The authors would like to acknowledge Olivero Andreussi, Marco Campetella and Giacomo Prampolini for useful discussions and CNPq for the financial support.

\section{References:}

1. Blankenship, R. E. Early evolution of photosynthesis. Plant Physiol. 154, 434-8 (2010).

2. Frisch, M. J. et al. Gaussian09, Revision A.1; Gaussian, Inc., Wallingford CT; (2009).

3. Barone, V. et al. Joyce and Ulysses: integrated and user-friendly tools for the parameterization of intramolecular force fields from quantum mechanical data. Phys. Chem. Chem. Phys. 15, 3736-51 (2013).

4. Van Der Spoel, D. et al. GROMACS: Fast, flexible, and free. J. Comput. Chem. 26, 1701-1718 (2005).

5. Curutchet, C. \& Mennucci, B. Quantum Chemical Studies of Light Harvesting. Chem. Rev. (2016). 